\title{
Multifractality of overlapping non-uniform self-similar measures
}

\author{
J. Neunhäuserer \\ neunchen@aol.com
}

\begin{abstract}
We prove that non-uniform self-similar measures have a multifractal spectrum in a parameter domain where the open set condition fails to hold.
\end{abstract}

MSC 2010: 28A80

Key-words: self-similar measures, multifractal analysis, dimension spectrum, local dimension, potential theory, transversality

\section{Introduction}

For a Borel probability measure $\mu$ on the real line $\mathbb{R}$, the local dimension of $\mu$ in $x$ is given by

$$
d(\mu, x)=\lim _{r \longmapsto 0} \frac{\log (\mu((x-r, x+r)))}{\log (r)},
$$

provided the limit exists. The multifractal analysis for the local dimension is concerned with the study of the level sets

$$
\Delta_{\mu}(\alpha)=\{x \mid d(x, \mu)=\alpha\}
$$

The goal is to determine or at least estimate the dimension spectrum

$$
f_{\mu}(\alpha)=\operatorname{dim}_{H}\left(\Delta_{\mu}(\alpha)\right)
$$

where $\operatorname{dim}_{H} A$ denotes the Hausdorff dimension of a set $A \subseteq \mathbb{R}$. The reader may consider $[1,2]$ or $[8]$ for an introduction to dimension theory. Measures that posses a non trivial dimension spectrum are called multifractal.

Definition 1.1 A Borel probability measure $\mu$ on $\mathbb{R}$ is a multifractal measures if $f_{\mu}(\alpha)>$ 0 holds for Lebesgue almost all $\alpha$ in some (non-degenerate) interval.

For $\beta_{0}, \beta_{1} \in(0,1)$ and $p \in(0,1)$ we consider in this paper non-uniform self-similar measures $\mu_{\beta_{0}, \beta_{1}}^{p}$ on $\mathbb{R}$ given by the relation

$$
\mu_{\beta_{0}, \beta_{1}}^{p}=p T_{0}\left(\mu_{\beta_{0}, \beta_{1}}^{p}\right)+(1-p) T_{1}\left(\mu_{\beta_{0}, \beta_{1}}^{p}\right)
$$

where $T_{i} x=\beta_{i}(x+i)$ for $i=0,1$. These measures exist and are known to be uniquely determined by the self-similarity relation, see [3]. If $\beta_{0}+\beta_{1} \leq 1$ the open set condition 
is fulfilled. This means that here is an open set $O \subseteq \mathbb{R}$ such that $T_{0}(O) \cup T_{1}(O) \subseteq O$ and $T_{0}(O) \cap T_{1}(O)=\emptyset$. Self-similar measures that fulfill the open set condition are know to be multifractal. It turns out that $f_{\mu}(\alpha)$ is the Legendre transform of the $L^{q}$ spectrum which is easy to compute for self-similar measures, see chapter 12 of [2]. In the case $\beta_{1}+\beta_{2}>1$ it follows from [5] that the measures $\mu_{\beta_{0}, \beta_{1}}^{p}$ are absolutely continuous (and even equivalent to the Lebesgue measure) for all $p \in(0,1)$ and almost all $\beta_{0}, \beta_{1} \in(0,0.6684)$ with $\beta_{0}^{p} \beta_{1}^{1-p}<p^{p}(1-p)^{1-p}$. Here we obtain the following result which especially holds for absolutely continuous $\mu_{\beta_{0}, \beta_{1}}^{p}$ :

Theorem 1.1 For all $p \in(0,1)$ and almost all $\beta_{0}, \beta_{1} \in(0,0.6684)$ with $\beta_{0}<p$ or $\beta_{1}<1-p$ the measure $\mu_{\beta_{0}, \beta_{1}}^{p}$ is multifractal.

Note that it is not all obvious that a self-similar measure which is absolutely continuous has a multifractal spectrum. In a resent paper of Jordan, Shmerkin and Solomyak [4] studied the multifractal structure of absolutely Bernoulli convolutions, which are uniform self-similar measures. In this paper Theorem 1.1 is proved in the special case $\beta=\beta_{1}=$ $\beta_{2} \in(0.5,0.6684)$. The proof is based on the potential theoretic method in dimension theory (see chapter 4 of [1]) and estimates using the transversality of the coding map (see $[6,7])$. In fact the curious bound 0.6684 is due to the transversality techniques used. This is a lower bound for the smallest double zeros of power series with coefficients bounded by one, see [9]. We adopt this approach here. It turned out that estimates on the potential are more delicate in the case of non-uniform self-similar measures (using the law of lagre numbers and uniform convergence on a set of large measure does not give the desires lower bound on the local dimension in our setting). We had to modify the potential theoretical approach and to refine estimates on the potential of the measures to realize their multifractal analysis. It should be possible to generalize our argument to self-similar given by $n$-different contractions also notations get somehow awkward in this setting.

The rest of the paper os organized as follows. In the next section we introduce some notations and present our main results. Then we describe the potential theoretical and transversality results we will use. The last section contains the proof of the main theorem.

\section{Notations and Results}

For $\beta_{0}, \beta_{1} \in(0,1)$ we introduce a description of self-similar measures by a symbolic coding $\pi_{\beta_{0}, \beta_{1}}:\{0,1\}^{\mathbb{N}} \mapsto \mathbb{R}$. Consider the map

$$
\pi_{\beta_{1}, \beta_{2}}\left(\left(s_{k}\right)\right)=\sum_{n=1}^{\infty} s_{n} \beta_{0}^{\mathbf{o}_{n}\left(\left(s_{k}\right)\right)} \beta_{1}^{\mathbf{1}_{n}\left(\left(s_{k}\right)\right)},
$$

where $\mathbf{0}_{n}\left(\left(s_{k}\right)\right)$ counts the zeros in $\left\{s_{1}, \ldots, s_{n}\right\}$ and $\mathbf{1}_{n}\left(\left(s_{k}\right)\right)$ counts the entries that are one. For $p \in(0,1)$ let $b^{p}$ be the Bernoulli measure $(p, 1-p)$ on $\{0,1\}^{\mathbb{N}}$. A simple 
calculation shows that the projected measure $\pi_{\beta_{0}, \beta_{1}}\left(b^{p}\right)$ is self-similar with respect to the function system $\left(T_{0}, T_{1}\right)$, hence

$$
\mu_{\beta_{0}, \beta_{1}}^{p}=\pi_{\beta_{0}, \beta_{1}}\left(b^{p}\right)
$$

The Lyapunov exponent of $b^{p}$ with respect to the function system $\left(T_{0}, T_{1}\right)$ is given by

$$
\Xi_{\beta_{0}, \beta_{1}}(p)=-p \log \left(\beta_{0}\right)-(1-p) \log \left(\beta_{1}\right)
$$

and the entropy of Bernoulli measures measure $b^{p}$ is well known to be

$$
H(p)=-p \log (p)-(1-p) \log (1-p)
$$

In addition we will use the condition entropy

$$
H(p, q)=-q \log (p)-(1-q) \log (1-p)
$$

for $q, p \in(0,1)$. We are now ready to state our main result on the local dimension $d\left(\mu_{\beta_{0}, \beta_{1}}^{p}, x\right)$ of non-uniform self-similar measure $\mu_{\beta_{0}, \beta_{1}}^{p}$.

Theorem 2.1 For all $p, q \in(0,1)$ and almost all $\beta_{0}, \beta_{1} \in(0,0.6684)$ with $H(p, q)<$ $\Xi_{\beta_{0}, \beta_{1}}(q)$ we have

$$
d\left(\mu_{\beta_{0}, \beta_{1}}^{p}, x\right)=\frac{H(p, q)}{\Xi_{\beta_{0}, \beta_{1}}(q)},
$$

for $\mu_{\beta_{0}, \beta_{1}}^{q}$-almost all $x \in \mathbb{R}$.

We will prove this result in section four. Theorem 2.1 has the following consequence:

Corollary 2.1 For all $p \in(0,1)$ and almost all $\beta_{0}, \beta_{1} \in(0,0.6684)$ with $H(p)<\Xi_{\beta_{0}, \beta_{1}}(p)$

$$
\operatorname{dim}_{H} \mu_{\beta_{0}, \beta_{1}}^{p}=\frac{H(p)}{\Xi_{\beta_{0}, \beta_{1}}(p)} .
$$

Proof. Setting $p=q$ in Theorem 2.1 we see that the local dimension of the measure $\mu_{\beta_{0}, \beta_{1}}^{p}$ is constant almost everywhere. Such a measure is called exact dimensional and it is known that all notations of dimension coincide. Especially the Hausdorff dimension of the measure is given by the local dimension, see [10].

In the case $\beta_{1}+\beta_{2} \leq 1$ this result is well known, see [2]. In the overlapping case $\beta_{1}+\beta_{2}>1$ we proved in [5] that $H(p) / \Xi_{\beta_{0}, \beta_{1}}(p)$ is a upper bound on the Hausdorff dimension of the measures. The identity for almost all parameters in a parameter domain is a new result.

Theorem 2.1 in combination with its corollary allows us to realize the multifractal analysis of some overlapping non-uniform self-similar measures. We obtain a lower bound on the Hausdorff dimension of the level sets $\Delta_{\mu_{\beta_{0}, \beta_{1}}^{p}}(\alpha)$, defined in the introduction. 
Theorem 2.2 For all $p \in(0,1)$, almost all $\beta_{0}, \beta_{1} \in(0,0.6684)$ and almost all $\alpha \in \mathbb{R}$ with

$$
\min \left\{\frac{\log (p)}{\log \left(\beta_{0}\right)}, \frac{\log (1-p)}{\log \left(\beta_{1}\right)}\right\} \leq \alpha \leq \min \left\{1, \max \left\{\frac{\log (p)}{\log \left(\beta_{0}\right)}, \frac{\log (1-p)}{\log \left(\beta_{1}\right)}\right\}\right\}
$$

we have

$$
f_{\mu_{\beta_{0}, \beta_{1}}^{p}}(\alpha)=\operatorname{dim}_{H} \Delta_{\mu_{\beta_{0}, \beta_{1}}^{p}}(\alpha) \geq \frac{H(q)}{\Xi_{\beta_{0}, \beta_{1}}(q)}>0,
$$

where $q \in(0,1)$ is determined by the relation $H(p, q)=\alpha \Xi_{\beta_{0}, \beta_{1}}(q)$.

Proof. Using Theorem 2.1 and the theorem of Fubini we have for all $p \in(0,1)$ and almost all $\beta_{0}, \beta_{1} \in(0,0.6684)$

$$
\mu_{\beta_{0}, \beta_{1}}^{q}\left(\left\{x \mid d\left(\mu_{\beta_{0}, \beta_{1}}^{p}, x\right)=\frac{H(p, q)}{\Xi_{\beta_{0}, \beta_{1}}(q)}\right\}\right)=1,
$$

for almost all $q \in(0,1)$ such that $H(p, q)<\Xi_{\beta_{0}, \beta_{1}}(q)$. Using Corollary 2.1 this implies

$$
\operatorname{dim}_{H} \Delta_{\mu}\left(\frac{H(p, q)}{\Xi_{\beta_{0}, \beta_{1}}(q)}\right)=\operatorname{dim}_{H}\left\{x \mid d\left(\mu_{\beta_{0}, \beta_{1}}^{p}, x\right)=\frac{H(p, q)}{\Xi_{\beta_{0}, \beta_{1}}(q)}\right\} \geq \operatorname{dim}_{H} \mu_{\beta_{0}, \beta_{1}}^{q}=\frac{H(q)}{\Xi_{\beta_{0}, \beta_{1}}(q)}
$$

for these $q \in(0,1)$. Now we set

$$
\alpha(q)=\frac{H(p, q)}{\Xi_{\beta_{0}, \beta_{1}}(q)} .
$$

Note that

$$
\max _{q \in(0,1)} \alpha(q)=\max \left\{\frac{\log (p)}{\log \left(\beta_{0}\right)}, \frac{\log (1-p)}{\log \left(\beta_{1}\right)}\right\} \text { and } \min _{q \in(0,1)} \alpha(q)=\min \left\{\frac{\log (p)}{\log \left(\beta_{0}\right)}, \frac{\log (1-p)}{\log \left(\beta_{1}\right)}\right\} .
$$

Thus the result follows immediately by changing variables.

Theorem 1.1 stated in the introducing follows easily from Theorem 2.2.

Proof of Theorem 1.1.Under the assumption $\beta_{0}<p$ or $\beta_{1}<1-p$ of Theorem 1.1

$$
\left[\min \left\{\frac{\log (p)}{\log \left(\beta_{0}\right)}, \frac{\log (1-p)}{\log \left(\beta_{1}\right)}\right\}, \min \left\{1, \max \left\{\frac{\log (p)}{\log \left(\beta_{0}\right)}, \frac{\log (1-p)}{\log \left(\beta_{1}\right)}\right\}\right]\right.
$$

is an interval and it is non-degenerate if $\log (p) \log \left(\beta_{1}\right) \neq \log (1-p) \log \left(\beta_{0}\right)$. But this hold for almost all $\beta_{1}, \beta_{2} \in(0,0.6684)$ (in the sense of two dimensional Lebesgue measure).

\section{Potential theoretic method and transversality}

We apply the potential theoretical method to obtain a lower bound on the local dimension of self-similar measures. We will use the following modification of a well know result in the proof of Theorem 2.1. 
Proposition 3.1 Let $\mu$ and $\nu$ be Borel probability measures on $\mathbb{R}$. If there exists $\epsilon>0$ such that

$$
\int\left(\int|x-y|^{-d} d \mu(y)\right)^{\epsilon} d \nu(x)<\infty
$$

for some $d=d(\epsilon)>0$ we have

$$
\underline{d}(\mu, x):=\liminf _{r \longmapsto 0} \frac{\log (\mu((x-r, x+r)))}{\log (r)} \geq d
$$

for $\nu$-almost all $x \in \mathbb{R}$.

Proof. Under the assumption we have

$$
\int|x-y|^{-d} d \mu(y)<\infty
$$

for $\nu$-almost all $x$. But for these $x$

$$
\frac{\mu((x-r, x+r)))}{r^{d}}
$$

remains bounded for $r \rightarrow 0$, hence $\underline{d}(\mu, x) \geq d$

The next proposition is based on the transversality properties of the coding map for non uniform self-similar measures introduced in the last section. It will be crucial in the next section to apply Proposition 3.1 in our context.

Proposition 3.2 There is a constant $B>0$ such that for all $\left(s_{k}\right),\left(t_{k}\right) \in\{0,1\}^{\mathbb{N}}, \beta_{0} \in$ $(0,0.6684), c \in(0,1]$ and $d \in(0,1)$ we have

$$
\int_{\beta_{0}}^{0.6684}\left|\pi_{\beta, c \beta}\left(\left(s_{k}\right)\right)-\pi_{\beta, c \beta}\left(\left(t_{k}\right)\right)\right|^{-d} d \beta \leq B \beta_{0}^{-d \mathbf{0}_{\mathfrak{n}}\left(\left(s_{k}\right)\right)}\left(c \beta_{0}\right)^{-d\left(1+\mathbf{1}_{\mathfrak{n}}\left(\left(t_{k}\right)\right)\right)},
$$

where $\mathfrak{n}=\mathfrak{n}\left(\left(s_{k}\right),\left(t_{k}\right)\right)=\max \left\{k \mid s_{i}=t_{i} \forall i \leq k\right\}$.

Proof. We know from Theorem 2.6 of [9] that there is a constant $B>0$ such that

$$
\mathfrak{L}\left\{\beta \in\left[\beta_{0}, 0.6684\right]|| \phi(\beta) \mid<r\right\} \leq B r .
$$

for all $r>0$ and all power series of the form

$$
\phi(x)=\sum_{k=1}^{\infty} a_{k} \beta^{k} \quad(\star)
$$

with $\left|a_{1}\right|=1$ and $\left|a_{k}\right| \leq 1$. This is due to the fact smallest double zero $\alpha \in(0,1)$ of a power series of this form is in the interval $(0.6684,0.6685)$ and the powers series hence intersect the axis transversally. A simple calculation performed in [5] shows

$$
\pi_{\beta, c \beta}\left(\left(s_{k}\right)\right)-\pi_{\beta, c \beta}\left(\left(t_{k}\right)\right)=\beta^{\mathbf{0}_{\mathfrak{n}}\left(\left(s_{k}\right)\right)}(c \beta)^{\left(1+\mathbf{1}_{\mathfrak{n}}\left(\left(t_{k}\right)\right)\right.} \phi_{\left(s_{k}\right),\left(t_{k}\right)}(\beta),
$$

where $\phi_{\left(s_{k}\right),\left(t_{k}\right)}(\beta)$ is a power series of the form $(\star)$. So the estimate on the integral is just a corollary to 2.6 of [9]. 


\section{Proof of theorem 2.1}

We split up the proof of theorem 2.1 into two steps. The proof of upper bound on the local dimension of measures is rather standard.

Proposition 4.1 For all $p, q \in(0,1)$ and all $\beta_{0}, \beta_{1} \in(0,1)$ with $H(p, q)<\Xi_{\beta_{0}, \beta_{1}}(q)$ we have

$$
\bar{d}\left(\mu_{\beta_{0}, \beta_{1}}^{p}, x\right):=\limsup _{r \longmapsto 0} \frac{\log \left(\mu_{\beta_{0}, \beta_{1}}^{p}((x-r, x+r))\right)}{\log (r)} \leq \frac{H(p, q)}{\Xi_{\beta_{0}, \beta_{1}}(q)}
$$

for $\mu_{\beta_{0}, \beta_{1}}^{q}$-almost all $x \in \mathbb{R}$.

Proof. For $\beta_{0}, \beta_{1} \in(0,1)$ consider the metric $\delta_{\beta_{0}, \beta_{1}}$ on $\{0,1\}^{\mathbb{N}}$ given by

$$
\delta_{\beta_{0}, \beta_{1}}\left(\left(s_{k}\right),\left(t_{k}\right)\right)=\beta_{0}^{\mathbf{0}_{\mathfrak{n}}\left(\left(s_{k}\right)\right)} \beta_{1}^{\mathbf{1}_{\mathfrak{n}}\left(\left(t_{k}\right)\right)},
$$

where $\mathfrak{n}=\mathfrak{n}\left(\left(s_{k}\right),\left(t_{k}\right)\right)=\max \left\{k \mid s_{k}=t_{k}\right\}$. Let $|A|_{\beta_{0}, \beta_{1}}$ be the diameter of $A \subseteq\{0,1\}^{\mathbb{N}}$ and let $\left[s_{1}, \ldots, s_{n}\right] \subseteq\{0,1\}^{\mathbb{N}}$ be a cylinder set. Using the law of large numbers we have

$$
\lim _{n \longmapsto \infty} \frac{\log \left(b^{p}\left(\left[s_{1}, \ldots, s_{n}\right]\right)\right)}{\log \left(\left|\left[s_{1}, \ldots, s_{n}\right]\right|_{\beta_{0}, \beta_{1}}\right)}=\lim _{n \longmapsto \infty} \frac{\log \left(p^{\mathbf{0}_{n}\left(\left(s_{k}\right)\right)}(1-p)^{\left.\mathbf{1}_{n\left(\left(s_{k}\right)\right)}\right)}\right.}{\log \left(\beta_{0}^{\mathbf{0}_{n}\left(\left(s_{k}\right)\right)} \beta_{1}^{\mathbf{1}_{n\left(\left(s_{k}\right)\right)}}\right)}=\frac{H(p, q)}{\Xi_{\beta_{0}, \beta_{1}}(q)}
$$

for $b^{q}$-almost all sequences $\left(s_{k}\right) \in\{0,1\}^{\mathbb{N}}$. The rest of the proof works along the same line as the proof Theorem I in [5]. We will not repeat the details here, one just shows that the coding map $\pi_{\beta_{0}, \beta_{1}}$ is Lipschitz with respect to the metric $\delta_{\beta_{0}, \beta_{1}}$ and Lipschitz maps do not increase dimension.

To prove the lower bound on the local dimension we will use the following technical proposition based on the transversally result in proposition 3.2.

Proposition 4.2 For all $p, q \in(0,1), \epsilon, c \in(0,1], \beta_{0} \in(0,0.6684)$ and $d \in(0,1)$ with

$$
\left(\frac{p^{\epsilon} q}{\beta_{0}^{d \epsilon}}+\frac{(1-p)^{\epsilon}(1-q)}{\left(c \beta_{0}\right)^{d \epsilon}}\right)^{1 / \epsilon}<1
$$

we have

$$
I:=\int_{\beta_{0}}^{0.6684} \int\left(\int|x-y|^{-d} d \mu_{\beta, c \beta}^{p}(x)\right)^{\epsilon} d \mu_{\beta, c \beta}^{q}(y) d \beta<\infty .
$$

Proof. Changing the order of integration and applying Hölder inequality, $\int f^{\epsilon} \leq C\left(\int f\right)^{\epsilon}$ for some constant $C$, we have

$$
I \leq C \int\left(\iint_{\beta_{0}}^{0.6684}|x-y|^{-d} d \beta d \mu_{\beta, c \beta}^{p}(x)\right)^{\epsilon} d \mu_{\beta, c \beta}^{q}(y) .
$$

Now changing variables using $\mu_{\beta_{0}, \beta_{1}}^{p}=\pi_{\beta_{0}, \beta_{1}}\left(b^{p}\right)$ we obtain

$$
I \leq C \int_{\{0,1\}^{\mathbb{N}}}\left(\int_{\{0,1\}^{\mathbb{N}}} \int_{\beta_{0}}^{0.6684}\left|\pi_{\beta, c \beta}\left(\left(s_{k}\right)\right)-\pi_{\beta, c \beta}\left(\left(t_{k}\right)\right)\right|^{-d} d \beta d b^{p}\left(\left(s_{k}\right)\right)\right)^{\epsilon} d b^{q}\left(\left(t_{k}\right)\right) .
$$


Using Proposition 3.2 and integrating the first time we have

$$
\begin{gathered}
I \leq C B^{\epsilon}\left(c \beta_{0}\right)^{-d \epsilon} \int_{\{0,1\}^{\mathbb{N}}}\left(\int_{\{0,1\}^{\mathbb{N}}} \beta_{0}^{-d \mathbf{0}_{\mathfrak{n}}\left(\left(s_{k}\right)\right)}\left(c \beta_{0}\right)^{-d \mathbf{1}_{\mathfrak{n}}\left(\left(t_{k}\right)\right)} b^{p}\left(\left(s_{k}\right)\right)\right)^{\epsilon} d b^{q}\left(\left(t_{k}\right)\right) \\
=C B^{\epsilon}\left(c \beta_{0}\right)^{-d \epsilon} \int_{\{0,1\}^{\mathbb{N}}}\left(\sum_{n=0}^{\infty} \beta_{0}^{-d \mathbf{0}_{n}\left(\left(t_{k}\right)\right)}\left(c \beta_{0}\right)^{-d \mathbf{1}_{n}\left(\left(t_{k}\right)\right)} b^{p}\left\{\left(s_{k}\right) \mid \mathfrak{n}\left(\left(s_{k}\right),\left(t_{k}\right)\right)=n\right\}\right)^{\epsilon} d b^{q}\left(\left(t_{k}\right)\right) \\
=C B^{\epsilon}\left(c \beta_{0}\right)^{-d \epsilon} \int_{\{0,1\}^{\mathbb{N}}}\left(\sum_{n=0}^{\infty}\left(\frac{p}{\beta_{0}^{d}}\right)^{\mathbf{0}_{n}\left(\left(t_{k}\right)\right)}\left(\frac{(1-p)}{\left(c \beta_{0}\right)^{d}}\right)^{\mathbf{1}_{n}\left(\left(t_{k}\right)\right)}\right)^{\epsilon} d b^{q}\left(\left(t_{k}\right)\right)
\end{gathered}
$$

With the inequality $\left(\sum x_{i}\right)^{\epsilon} \leq\left(\sum x_{i}^{\epsilon}\right)$ and integrating again we have

$$
\begin{gathered}
I \leq C B^{\epsilon}\left(c \beta_{0}\right)^{-d \epsilon}\left(1+\sum_{n=1}^{\infty} \sum_{\left(t_{1}, \ldots t_{n}\right) \in\{0,1\}^{n}}\left(\frac{p^{\epsilon} q}{\beta_{0}^{d \epsilon}}\right)^{\mathbf{0}_{n}\left(\left(t_{k}\right)\right)}\left(\frac{(1-p)^{\epsilon}(1-q)}{\left(c \beta_{0}\right)^{d \epsilon}}\right)^{\mathbf{1}_{n}\left(\left(t_{k}\right)\right)}\right) \\
=C B^{\epsilon}\left(c \beta_{0}\right)^{-d \epsilon}\left(1+\sum_{n=1}^{\infty}\left(\frac{p^{\epsilon} q}{\beta_{0}^{d \epsilon}}+\frac{(1-p)^{\epsilon}(1-q)}{\left(c \beta_{0}\right)^{d \epsilon}}\right)^{n}\right)
\end{gathered}
$$

The geometric series converges by assumption.

Proof of Theorem 2.1. Combining Proposition 3.1 and 4.1 we have:

For all $p, q \in(0,1), \epsilon, c \in(0,1]$ and almost all $\beta_{0} \in(0,0.6684)$ and $d=d(\epsilon)$ with

$$
\left(\frac{p^{\epsilon} q}{\beta_{0}^{d \epsilon}}+\frac{(1-p)^{\epsilon}(1-q)}{\left(c \beta_{0}\right)^{d \epsilon}}\right)^{1 / \epsilon}=1-\epsilon
$$

$\underline{d}\left(\mu_{\beta_{0}, c \beta_{0}}^{p}, x\right) \geq d$ for $\mu_{\beta_{0}, c \beta_{0}}^{q}$-almost all $x \in \mathbb{R}$. Note that

$$
\lim _{\epsilon \longmapsto 0}\left(\frac{p^{\epsilon} q}{\beta_{0}^{d \epsilon}}+\frac{(1-p)^{\epsilon}(1-q)}{\left(c \beta_{0}\right)^{d \epsilon}}\right)^{1 / \epsilon}=\left(\frac{p}{\beta_{0}^{d}}\right)^{q}\left(\frac{1-p}{\left(c \beta_{0}\right)^{d}}\right)^{1-q}
$$

hence

$$
\lim _{\epsilon \longmapsto 0} d(\epsilon)=H(p, q) / \Xi_{\beta_{0}, c \beta_{0}}(q)<1
$$

since $H(p, q)<\Xi_{\beta_{0}, c \beta_{0}}(q)$ by the assumption of Theorem 2.1. Consequently for all $p, q \in$ $(0,1), c \in(0,1]$ and almost all $\beta_{0} \in(0,0.6684)$ we have $\underline{d}\left(\mu_{\beta_{0}, c \beta_{0}}^{p}, x\right) \geq H(p, q) / \Xi_{\beta_{0}, c \beta_{0}}(q)$, and by the virtue of Proposition 4.1 even $d\left(\mu_{\beta_{0}, c \beta_{0}}^{p}, x\right)=H(p, q) / \Xi_{\beta_{0}, c \beta_{0}}(q)$, for $\mu_{\beta_{0}, c \beta_{0}}^{q}$ almost all $x \in \mathbb{R}$. Theorem 2.1 follows by applying the theorem of Fubini.

\section{References}

[1] K.J. Falconer, Fractal Geometry - mathematical foundations and applications, Wiley, New York, 1990. 
[2] K.J. Falconer, Techniques in fractal geometry, Wiley and Sons, Chichester, 1997.

[3] J.J. Hutchinson, Fractals and self-similarity, Indiana Univ. Math. J. 30, 271-280, 1981.

[4] T. Jordan, P. Shmerkin and B. Solomyak, Multifractal structure of Bernoulli convolutions, Math. Proceedings of the Cambridge Philosophical Society. 151(3), 521-529, 2011.

[5] J. Neunhäuserer, Properties of some overlapping self-similar and some self-affine measures, Acta Mathematica Hungarica, vol. 92 (1-2), 143-161, 2001.

[6] Y. Peres and B. Solomyak, Absolutely continuous Bernoulli convolutions - a simple proof, Math. Research Letters 3, no. 2, 231-239, 1996.

[7] Y. Peres and B. Solomyak, Self-similar measures and intersection of Cantor sets, Trans. Amer. Math. Soc 350, no. 10, 4065-4087, 1998

[8] Ya. Pesin, Dimension Theory in Dynamical Systems - contemporary views and applications, University of Chicago Press, Chicago, 1997.

[9] P. Shmerkin and B. Solomyak, Zeros of $\{-1,0,1\}$ power series and connectedness loci of self-ane sets, Experimental Math. 15, no. 4, 499-511, 2006.

[10] L.S. Young, Dimension, entropy and Lyapunov exponents, Ergod. Thy. Dyn. Sys. 2, 109-124, 1982. 